Supplementary Information

\title{
Perovskite Color Detectors: Approaching the Efficiency Limit
}

Mohammad Ismail Hossain ${ }^{1,2}$, Haris Ahmad Khan ${ }^{3}$, Masayuki Kozawa4 ${ }^{4}$, Wayesh Qarony $^{1}$, Alberto Salleo ${ }^{5}$, Jon Yngve Hardeberg ${ }^{6}$, Hiroyuki Fujiwara ${ }^{4}$, Yuen Hong Tsang ${ }^{*}, 1,2$, Dietmar Knipp ${ }^{*}, 5$

1.) Department of Applied Physics and Materials Research Center, The Hong Kong Polytechnic University, Hung Hom, Kowloon, Hong Kong

2.) Shenzhen Research Institute, The Hong Kong Polytechnic University, 518057 Shenzhen, China

3.) Farm Technology Group, Wageningen University \& Research, Wageningen, The Netherlands

4.) Department of Electrical, Electronic and Computer Engineering, Gifu University, Gifu, Japan

5.) Geballe Laboratory for Advanced Materials, Department of Materials Science and Engineering, Stanford University, Stanford, USA

6.) The Norwegian Colour and Visual Computing Laboratory, NTNU-Norwegian University of Science and Technology, Gjøvik, Norway *Corresponding authors: yuen.tsang@polyu.edu.hk (Y.H. Tsang)

dknipp@stanford.edu (D. Knipp) 
Table S1: Layer sequence of the stacked perovskite color sensors.

\begin{tabular}{|c|c|c|c|}
\hline Function & Material & Thickness & Optical constants \\
\hline UV blocking layer & $\mathrm{MAPbCl}_{3}$ & $500 \mathrm{~nm}$ & Fig. S1 \\
\hline Contact top diode & Doped $\mathrm{ZnO}$ & $75 \mathrm{~nm}$ & Ref. [24,28] \\
\hline Absorber top diode & $\mathrm{MAPb}\left(\mathrm{Br}_{0.63} \mathrm{Cl}_{0.37}\right)_{3}$ & $500 \mathrm{~nm}$ & Fig. S1 \\
\hline $\begin{array}{c}\text { Contact top and } \\
\text { middle diode }\end{array}$ & Doped $\mathrm{ZnO}$ & $75 \mathrm{~nm}$ & Ref. [24,28] \\
\hline $\begin{array}{c}\text { Absorber middle } \\
\text { diode }\end{array}$ & $\left(\mathrm{MAPbl}_{0.2} \mathrm{Br}_{0.8}\right)_{3}$ & $700 \mathrm{~nm}$ & Fig. S1 \\
\hline $\begin{array}{c}\text { Contact middle } \\
\text { and bottom diode }\end{array}$ & Doped $\mathrm{ZnO}$ & $75 \mathrm{~nm}$ & Fig. S1 \\
\hline $\begin{array}{c}\text { Absorber bottom } \\
\text { diode }\end{array}$ & $\mathrm{MAPb}\left(\mathrm{I}_{0.6} \mathrm{Br}_{0.4}\right)_{3}$ & $1000 \mathrm{~nm}$ & Ref. [24,28] \\
\hline $\begin{array}{c}\text { Contact bottom } \\
\text { diode }\end{array}$ & Doped $\mathrm{ZnO}$ & $75 \mathrm{~nm}$ & Ref. [24] \\
\hline Reflector & $\mathrm{Al}$ & - & \\
\hline
\end{tabular}



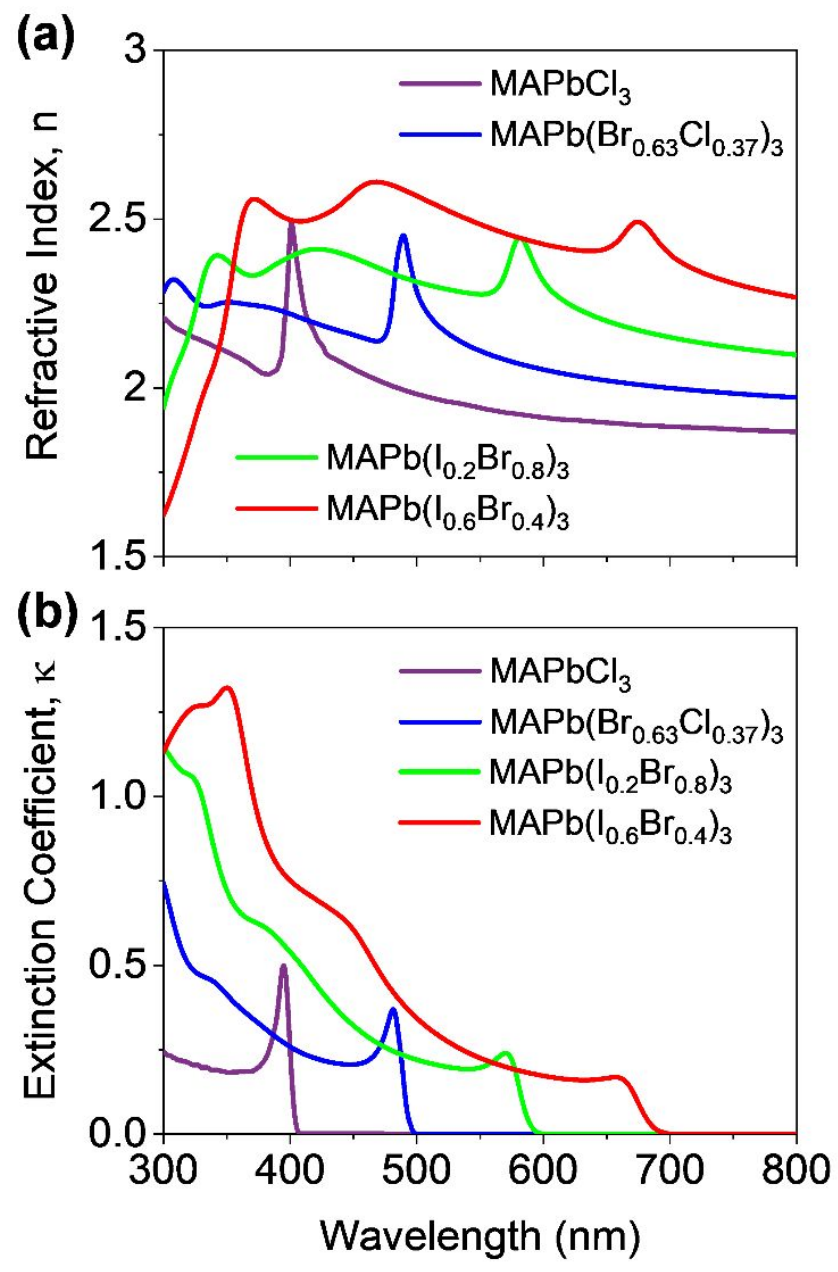

Figure S1: (a) Refractive index and (b) extinction coefficient of perovskite absorber materials used as UV blocking layer $\left(\mathrm{MAPbCl}_{3}\right)$, blue-channel $\left(\mathrm{MAPb}\left(\mathrm{Br}_{0.63} \mathrm{Cl}_{0.37}\right)_{3}\right)$, green-channel ( $\left.\mathrm{MAPb}\left(\mathrm{I}_{0.2} \mathrm{Br}_{0.8}\right)_{3}\right)$, and red-channel $\left(\mathrm{MAPb}\left(\mathrm{I}_{0.6} \mathrm{Br}_{0.4}\right)_{3}\right)$. 


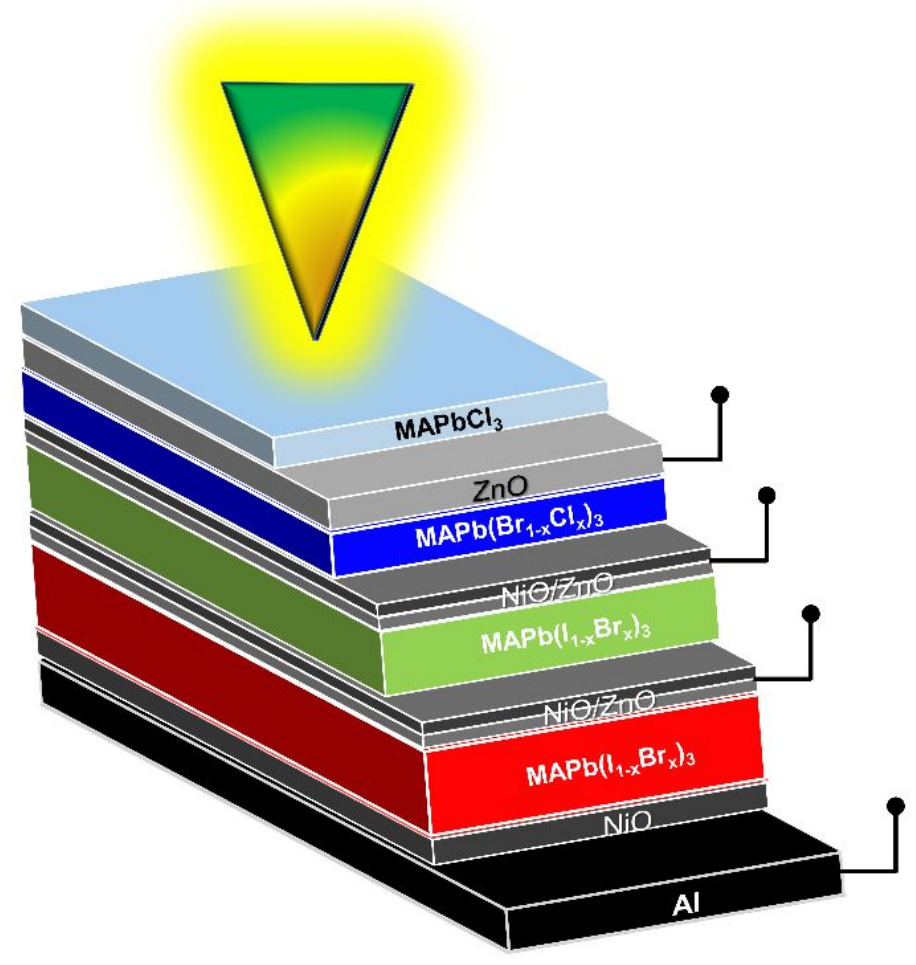

Figure S2: A schematic cross-section of the sensor, including the electrical interconnects to the external readout electronics.

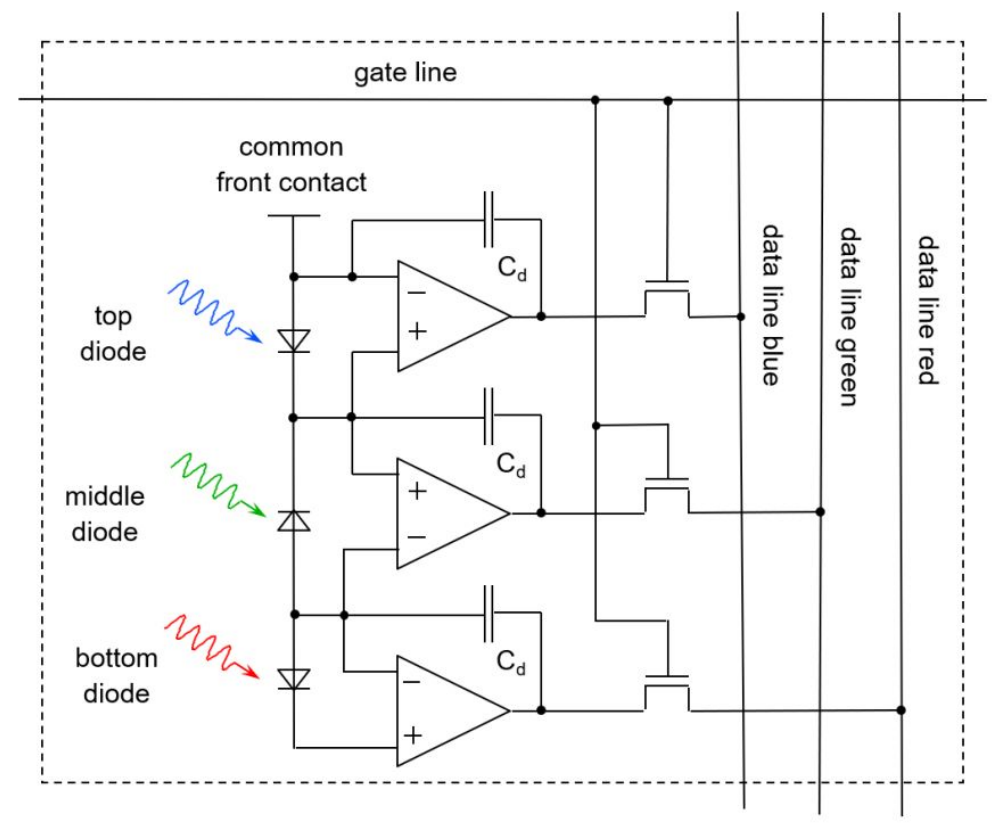

Figure S3: Pixel circuit of a vertically stacked color sensor consisting of three stacked diodes. Pixel circuit comparable to other CMOS based active pixel sensors. 


\section{Optical Modelling:}

In the first step the electric field distribution of the complete sensor structure is calculated including the UV blocking layer as shown in Fig. 4. Finite-difference time-domain (FDTD) in three dimensions were used to calculate the electric field distribution. Next, the power density distribution is calculated using the following equation,

$$
P(x, y, z)=\frac{1}{2} c \varepsilon_{0} n \alpha|E(x, y, z)|^{2}
$$

where $E$ is the electromagnetic field distribution, $c$ and $\varepsilon_{0}$ are speed of light and permittivity of free space, $\mathrm{n}$ is the refractive index of medium, and $\alpha$ is the absorption coefficient of material.

In the next step the absorption for each layer of the layer stack is calculated by

$$
A(\lambda)=\frac{1}{P_{\text {incident }}} \int P(x, y, z) d x d y d z .
$$

Only the photons absorbed by the perovskite layer will contribute to the photocurrent, while electron/hole pairs generated in the other layers of the sensor will be lost due to recombination. The quantum efficiency of the three sensor channels is calculated by

$$
Q E(\lambda)=A(\lambda) \times C E(\lambda)
$$

where $\operatorname{CE}(\lambda)$ is the collection efficiency, which is assumed to be $100 \%$. This is a reasonable assumption because the thickness of the perovskite absorbers is smaller than the diffusion lengths. Finally, the spectral responsivity is calculated by

$$
S R(\lambda)=\frac{q \lambda}{h c} Q E(\lambda)
$$

where $\mathrm{q}$ is the elementary charge, $\mathrm{h}$ is the Planck constant and $\mathrm{c}$ is the speed of light. The Full Width at half Maximum is extracted from the calculated spectral responsivities for the top, middle and bottom diode. 

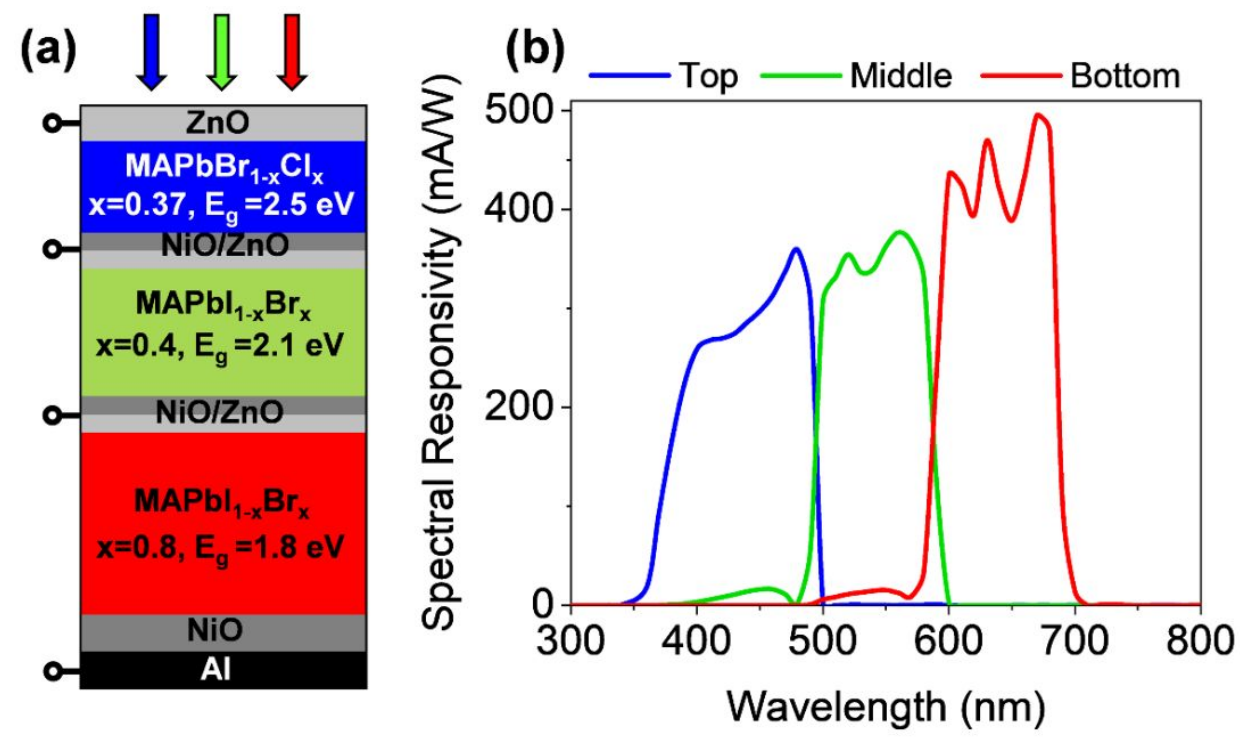

Figure S4: (a) Schematic cross-section of flat vertically stacked perovskite color sensor. (b) The simulated corresponding spectral responsivity of the flat vertically stacked perovskite color sensor.

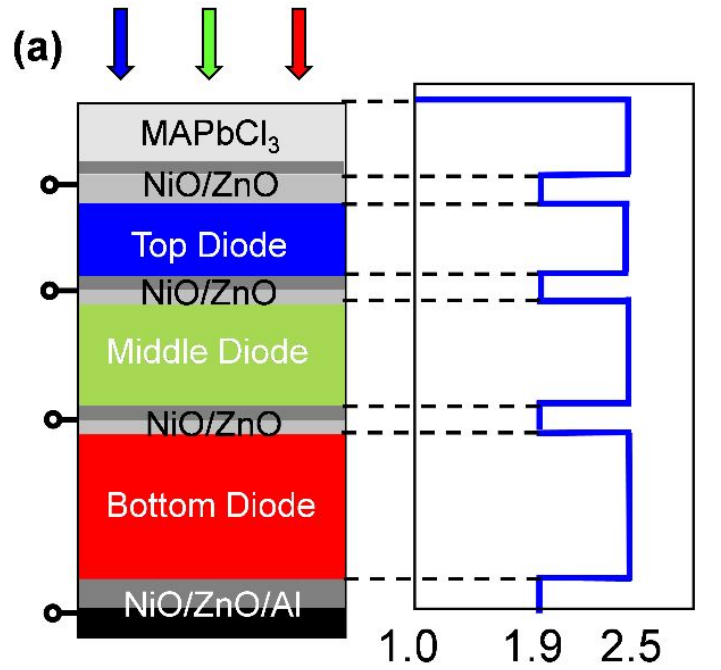

Effective Refractive Index

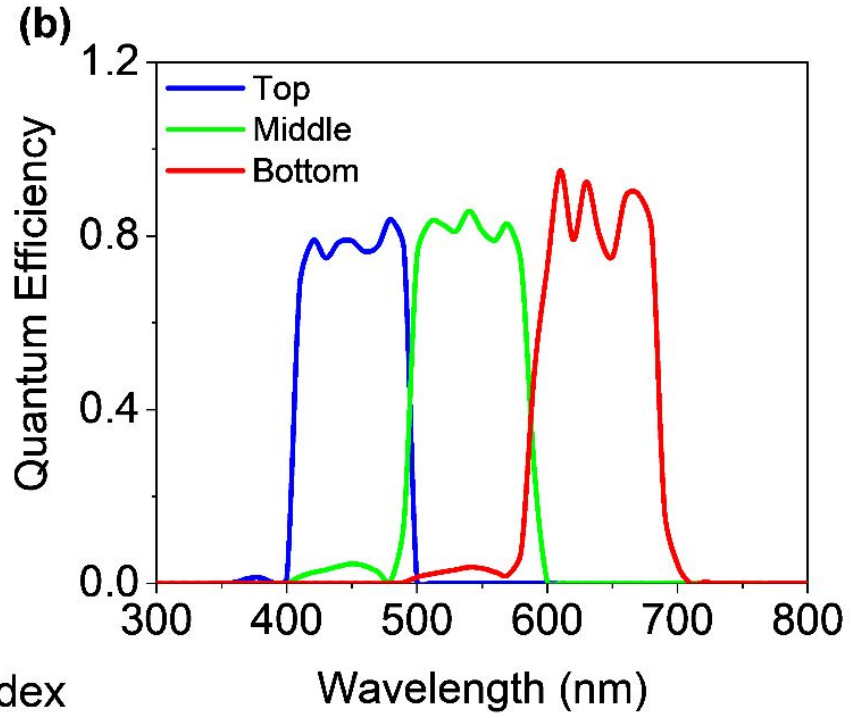

\begin{tabular}{ccc} 
Top Diode $\left(\mathrm{E}_{\mathrm{g}} \sim 2.5 \mathrm{eV}\right)$ & Middle Diode $\left(\mathrm{E}_{\mathrm{q}} \sim 2.1 \mathrm{eV}\right)$ & Bottom Diode $\left(\mathrm{E}_{\mathrm{g}} \sim 1.8 \mathrm{eV}\right)$ \\
$\operatorname{MAPb}\left(\mathrm{Br}_{0.63} \mathrm{Cl}_{0.37}\right)_{3}$ & $\operatorname{MAPb}\left(\mathrm{I}_{0.6} \mathrm{Br}_{0.4}\right)_{3}$ & $\operatorname{MAPb}\left(\mathrm{I}_{0.2} \mathrm{Br}_{0.8}\right)_{3}$ \\
\hline
\end{tabular}

Figure S5: (a) Schematic cross-section and corresponding effective refractive index of flat vertically stacked perovskite color sensor with a UV blocking layer. (b) The simulated corresponding quantum efficiency of the flat vertically stacked perovskite color sensor. 

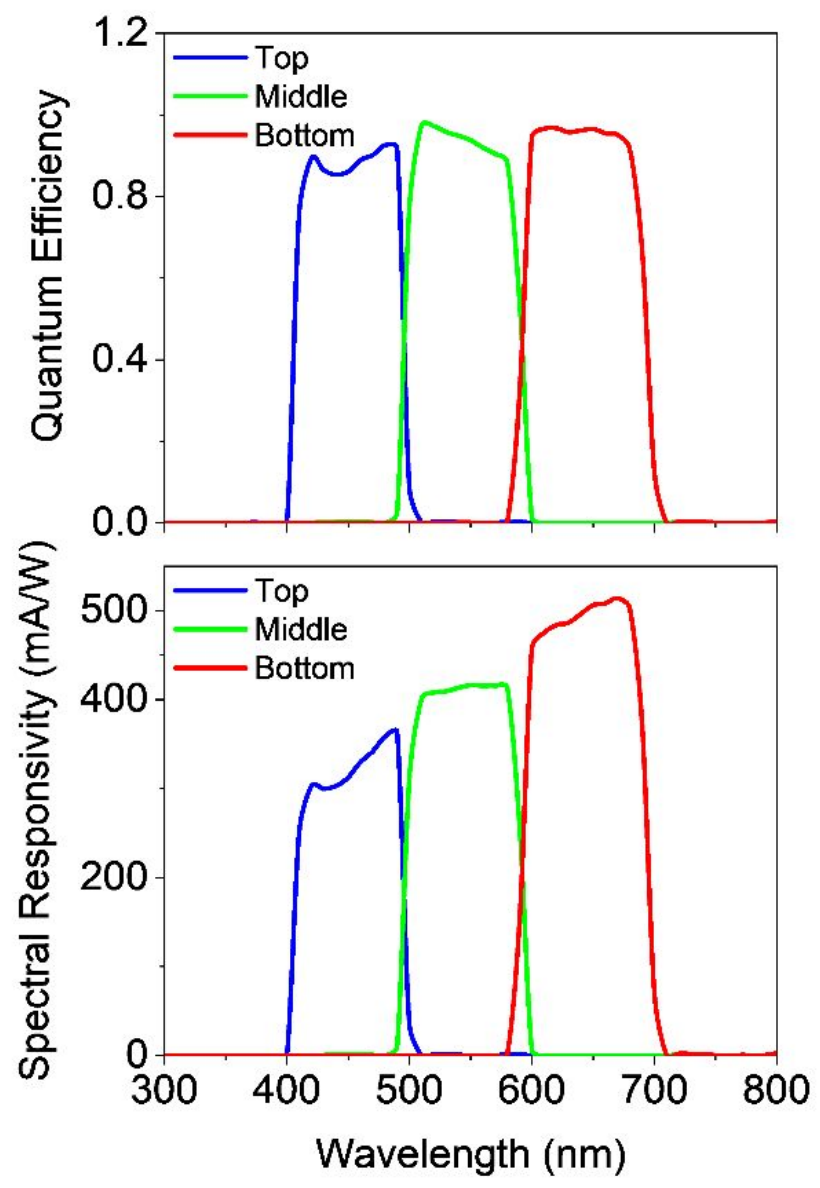

Figure S6: Simulated stacked color sensor with thicknesses of perovskite absorber layers larger than diffusion lengths of the carriers (UV blocking layer $900 \mathrm{~nm}$, absorber top diode $900 \mathrm{~nm}$, absorber middle diode $1500 \mathrm{~nm}$, absorber bottom diode $2000 \mathrm{~nm}$ ). The quantum efficiency and spectral response curves do not exhibit crosstalk. 
(a)

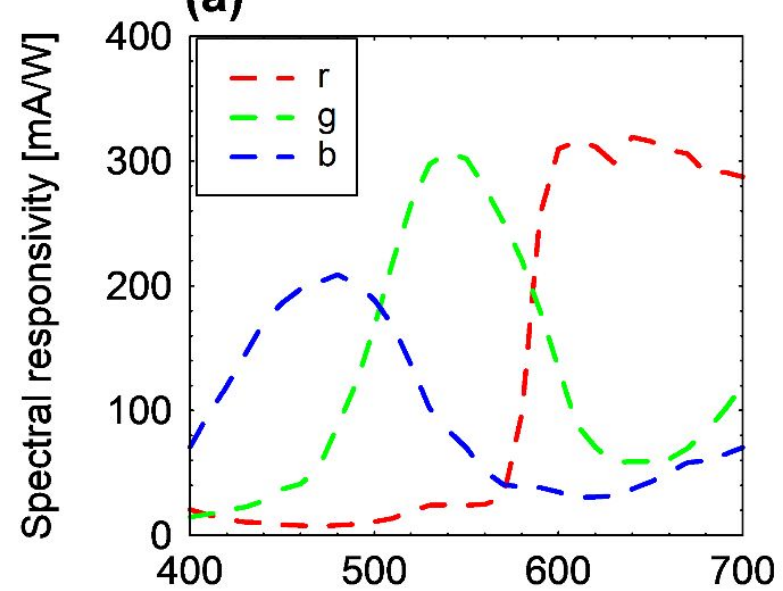

(c)

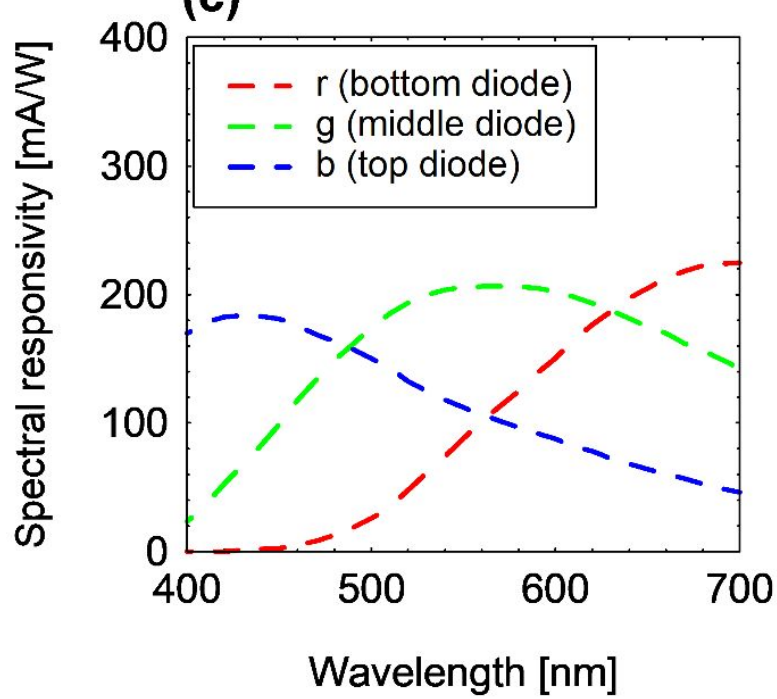

(b)

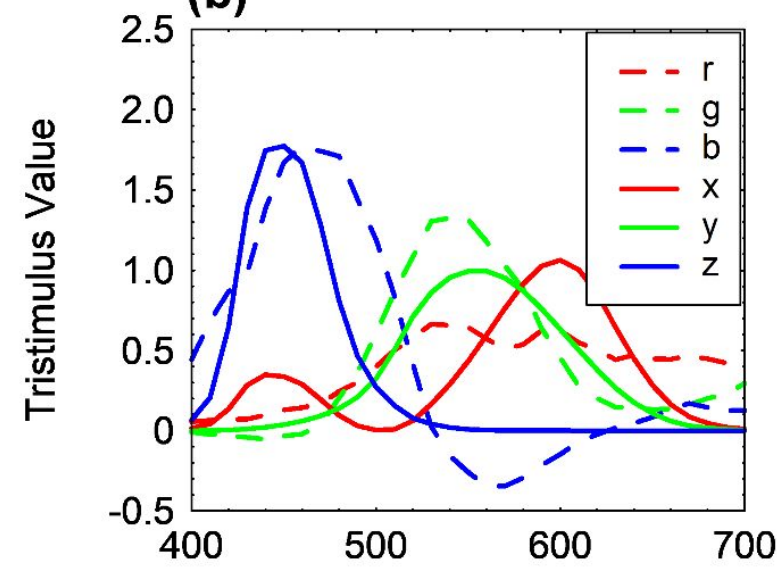

(d)

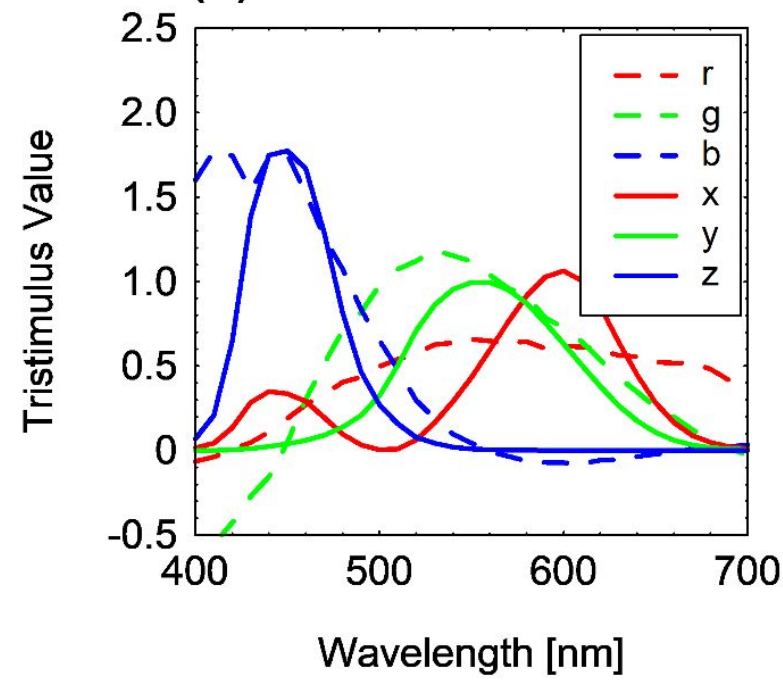

Figure S7: Measured spectral sensitivities of an optical sensor using a (a) side-by-side arrangement of optical filters and a (c) stacked silicon sensor (Foveon sensor). Standard CIE color-matching functions (xyz) and calculated color matching function (rgb) of the sensor using (b) optical filters and a (d) stacked silicon sensor (Foveon sensor). A linear color transformation was used. The standard CIE color-matching functions xyz represent the reference system. 